\title{
Ingresos por trabajo y esfuerzo laboral de los hogares en México durante la crisis económica, 2008-2010 1
}

\author{
María Valeria Judith Montoya García \\ Universidad Autónoma del Estado de Hidalgo
}

Recepción: 26 de julio de 2016 Aceptación: 28 de marzo de 2017

Resumen El objetivo de esta investigación es analizar los cambios en el esfuerzo laboral que debieran realizar los hogares en las zonas urbanas de México para mantener sus condiciones de vida ante el descenso de los ingresos por trabajo observados desde la irrupción de la crisis financiera de 20082009. Los principales resultados han mostrado que, a partir de la crisis económica, se registró una disminución en la mediana de los ingresos laborales reales por hora y un aumento en el promedio de horas dedicadas semanalmente al trabajo para el mercado, así como en el esfuerzo laboral. Pero los cambios presentados fueron distintos según la clase socioocupacional del hogar, pues tuvieron consecuencias más negativas para aquellos que ocupan su fuerza de trabajo de forma no asalariada.

PALABRAS CLAVE: esfuerzo laboral, ingresos laborales, hogares, trabajo, crisis económica.

Abstract The objective of this research is to analyze changes in work effort that households in urban areas of Mexico should make to maintain their living conditions with the decline in labor income observed since the outbreak of the financial crisis of 2008-2009. The main results showed that, from the economic crisis, a decline was recorded in the median of real labor income per hour and an increase in the average of hours dedicated weekly to work for the market as well as work effort. But the changes presented were different according to the socio-occupational class of the home, having more negative consequences for those who use their work force in a non-salaried way.

KEY WORDS: labor effort, labor income, households, work, economic crisis.

En esta investigación se utilizan los términos hogar y unidad doméstica para designar a los grupos que comparten una vivienda y un presupuesto común, y que además comparten vínculos de sangre, adopción o matrimonio. Por lo tanto, se excluye a los hogares compuestos y complejos. 
nte la presencia de crisis económicas de gran magnitud, como la aconte-
cida en el periodo 2008-2009, ha existido un mayor interés por investigar
sus efectos en los sectores productivos y las variables macroeconómicas. $\mathrm{Al}$ respecto, se ha encontrado que hubo una caída en las exportaciones, un decremento en la producción y un aumento en la inflación (Guillén, 2009; Mariña, 2012; Ortiz, 2012). En contraste, se ha abordado en menor medida lo que sucedió a nivel micro, específicamente con los hogares de las zonas urbanas de México, ante el aumento del desempleo y la caída de los ingresos laborales.

La relación entre familia y trabajo ha sido objeto de diversas investigaciones de tipo sociodemográfico, en las que sobresalen aquellas que dan cuenta de las respuestas de los hogares ante el descenso en sus condiciones de vida como consecuencia de las políticas de ajuste de la década de los ochenta del siglo pasado y las diversas crisis registradas posteriormente (Cortés \& Rubalcava, 1991; Tuirán, 1993; Estrada, 1999; García \& Pacheco, 2000).

El objetivo del presente artículo es analizar los cambios en el esfuerzo laboral que debieran realizar los hogares de las zonas urbanas de México para mantener sus condiciones de vida ante el descenso de los ingresos por trabajo que se registró a partir de la crisis financiera de 2008-2009 en el país. Se busca abonar al conocimiento del tema de la familia y el trabajo a partir de visibilizar las posibilidades que tienen los hogares de incrementar la oferta laboral en momentos de dificultades económicas generalizadas como medida para compensar los abruptos descensos de sus ingresos monetarios. La propuesta presentada toma como base los planteamientos teóricos de la economía política que establecen que la fuerza de trabajo necesita una serie de elementos para producirse y reproducirse, ya que es vista como una mercancía. Por esta razón se requiere un conjunto de satisfactores que permitan reponer la capacidad laboral del trabajador y de su familia.

En el primer apartado de este artículo se realiza una breve revisión de los cambios ocurridos en la economía mexicana a partir de la irrupción de la crisis financiera mundial reciente. La segunda sección muestra los efectos de la recesión económica en el mercado de trabajo, como el aumento del desempleo y la caída en la cantidad de puestos de trabajo formales. En la tercera se presenta la relación de los hogares con el mercado de trabajo por medio de una clasificación socioocupacional. En el siguiente apartado se hace un análisis de los ingresos laborales reales por hora de los hogares antes y después de la crisis. En la quinta sección se examina el esfuerzo laboral de los hogares, medido en horas de trabajo, y el que debieran realizar para acceder a un conjunto de bienes y servicios necesarios para la satisfacción de sus necesidades como forma de observar sus condiciones de vida. Finalmente, se exponen algunas reflexiones sobre los resultados de esta investigación. 
El análisis que se muestra a continuación se centra en las zonas urbanas de México (localidades de 2,500 habitantes y más). Para ello se utilizaron datos de la Encuesta Nacional de Ingresos y Gastos de los Hogares (ENIGH) para 2008 y 2010, con el fin de observar los cambios ocurridos a partir de la crisis económica en los ingresos de los hogares.

\section{Crisis financiera mundial y crisis de la economía mexicana}

Durante 2008 y 2009 México sufrió una de las peores crisis económicas, que comenzó en el sector financiero internacional y tuvo su génesis en Estados Unidos; el Fondo Monetario Internacional (FMI) la consideró como el "mayor shock financiero desde los treintas" (Guillén, 2009), al compararla con la Gran Depresión de 1929. Se manifestó desde principios de 2007, pero fue en marzo de 2008 cuando inició lo que sería una recesión de alcance mundial. Cámara y Mariña (2010), al hacer un análisis de la crisis financiera, encontraron su origen en la reestructuración neoliberal que tuvo el capitalismo a nivel mundial a partir de la década de los setenta del siglo pasado, que dio lugar al predominio relativo de las formas de valorización financiera y especulativa frente a las productivas. Asimismo, explican que no puede ser concebida como una crisis de la esfera financiera desligada de las tendencias estructurales de la rentabilidad y acumulación capitalista, dada la incapacidad del modelo neoliberal para reconstituir las condiciones de valorización de largo plazo del capital industrial. ${ }^{2}$

Si bien la crisis comenzó en el espacio financiero, se expandió a la economía real a través de las dificultades de pago de distintos agentes económicos, como deudores hipotecarios, empresas constructoras, consumidores, bancos comerciales, etc. (Guillén, 2009; Ortiz, 2012), lo que terminó por afectar la demanda agregada en distintos países.

Aunque la crisis financiera mostró sus primeras señales en 2007 en Estados Unidos, Mariña (2012) señala que transcurrieron algunos meses antes de que se pasara a la economía real de los países menos desarrollados. Para Guillén (2009), uno de los canales de transmisión de la crisis fue el relacionado con la lógica de la inversión de cartera; esta, ante la presencia de este tipo de fenómenos, se retira de los países emergentes y se refugia en mercados de capital más seguros. Explica que, a raíz de lo anterior, las monedas de Brasil, México y Chile sufrieron un embate especulativo no visto desde la crisis argentina de 2001.

2 Existe un debate, desde la perspectiva marxista, sobre las causas de la crisis. Para mayor información sobre la génesis y el desarrollo de ella y su interpretación teórica, véanse Guillén (2009), Boltvinik (2010), Cámara y Mariña (2010), Dumenil y Levy (2011) y Mariña (2012). 
Además, diversos investigadores (Guillén, 2009; Ocampo, 2009; Pérez Akaki, 2010; Mariña, 2012) encontraron que las principales vías de propagación de la crisis hacia Latinoamérica fueron la caída de los precios internacionales de las materias primas, la baja en las remesas y la disminución de los servicios turísticos, fuertemente dependientes de la demanda estadounidense.

Aun cuando la crisis económica mundial redujo las tasas de crecimiento del producto interno bruto (РІв) de los países latinoamericanos, algunos registraron tasas positivas, como Bolivia, Haití y Brasil. El caso mexicano fue sobresaliente porque registró su caída más abrupta en la producción: mientras que el decremento para toda la región latinoamericana fue de $1.8 \%$, para México fue cercana al $8 \%$ (CEPAL, 2009).

La profunda depresión que vivió el país tiene su causa en la reducción significativa en el volumen del comercio mundial, incluyendo los intercambios con Estados Unidos, país al que se dirige gran parte de las exportaciones manufactureras mexicanas. Pérez Akaki (2010) señala que las exportaciones de bienes cayeron entre el segundo trimestre de 2008 y el primero de 2009, lo cual provocó una fuerte contracción del PIB y la pérdida de miles de empleos.

Las diferencias en la profundidad de la crisis entre países se explican por las diversas formas de articulación de los mercados nacionales con el mercado mundial a partir de la puesta en marcha de las políticas neoliberales, así como por las estrategias de política económica implementadas para hacer frente a la crisis. $\mathrm{Al}$ respecto, Mariña (2012) indica que se ha observado que en México se han establecido grandes trasnacionales ligadas comercialmente con Estados Unidos, así como un modelo maquilador sin eslabonamiento productivo con la industria nacional, por lo que la generación de valor agregado es muy baja.

Para el presente artículo, la relevancia de la crisis en México se encuentra en los efectos que tuvo sobre la economía real, el empleo y las remuneraciones de los trabajadores. En cuanto a los hogares, tuvieron que hacer frente a condiciones más adversas en el mercado de trabajo, como un aumento en la desocupación y la precarización del empleo, que afectaron en forma desigual sus condiciones de vida.

\section{Efectos de la recesión económica sobre el mercado de trabajo urbano en México}

Para las unidades domésticas, las crisis económicas suelen representar un momento en que surgen o se acrecientan las carencias materiales debido a la disminución de los ingresos, principalmente por la pérdida del empleo de alguno de sus integrantes a consecuencia del aumento general en la desocupación. Damián (2012) señala que dicho fenómeno se concentra en las áreas urbanas porque es en ellas donde se aglutina la quiebra empresarial. Esta situación es relevante si se considera 
que, en promedio, tanto para 2008 como para 2010, el $90 \%$ de los ingresos monetarios de los hogares que participaban en el mercado laboral provenían de la venta de su fuerza de trabajo. ${ }^{3}$

Según información de la Encuesta Nacional de Ocupación y Empleo (ENOE) para las zonas más urbanizadas del país, en el tercer trimestre de 2009 se registraron las tasas de desocupación más altas desde la crisis de 1994. Para los hombres fue de $7.5 \%$ y para las mujeres de $8 \%$, después de una tendencia alcista iniciada a finales de 2007 (gráfica 1). Se debe destacar el aumento constante del desempleo en México desde el primer trimestre de 2003, en un contexto en que históricamente se han presentado tasas bajas, incluso del $2 \%$ a finales de la década de los noventa.

GRÁFICA 1. México, 1999-2010. Tasa de desocupación por sexo (zonas más urbanizadas de México) y tasa de crecimiento del PIB

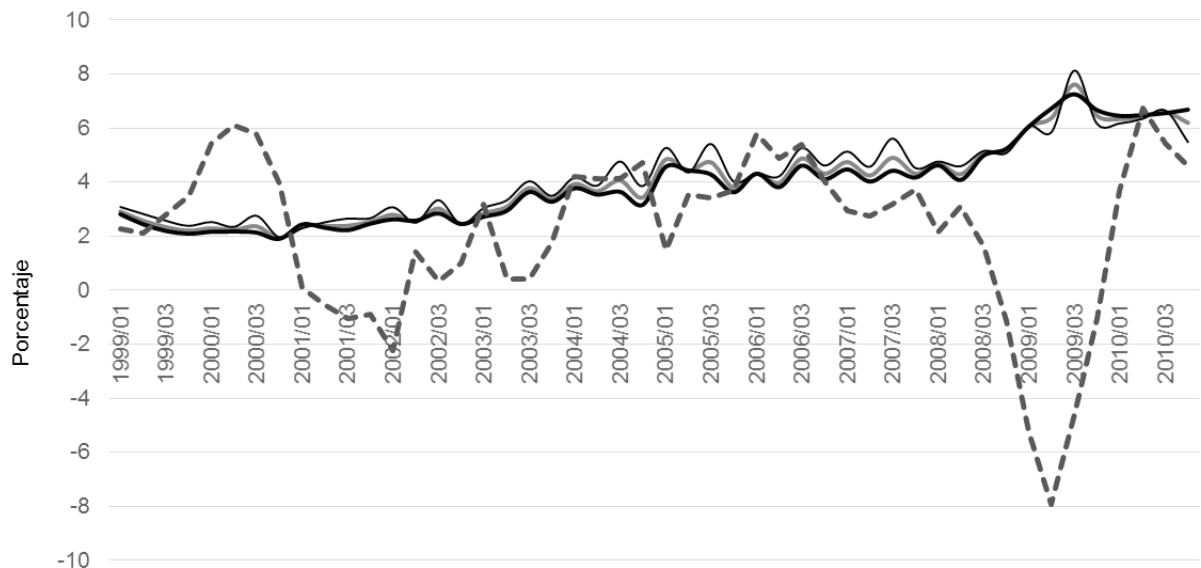

Año/Trimestre

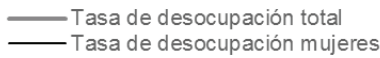

Tasa de desocupación hombres
$-\sim$ PIB

Nota: De 1999/01 a 2002/04 los datos corresponden a 48 ciudades. De 2002/01 a 2004/04, a 32; el cálculo se hizo con base en la población de 12 años y más. Ambas series fueron calculadas a partir de la Encuesta Nacional de Empleo Urbano (ENEU). De 2005/01 a 2010/04, los datos corresponden a 32 ciudades e incluye a la población de 14 y más años; la serie se calculó con base en los datos captados por la ENOE. Fuente: Elaboración propia a partir del Banco de Información Económica (BIE) y del sistema de Consulta Interactiva de Datos del INEGI.

El comportamiento de la tasa de desocupación ha estado desvinculado de la tasa de crecimiento del PIB, que para el primer trimestre de 2006 y 2007 alcanzó tasas superiores al $3 \%,{ }^{4}$ mientras que las de desempleo fueron del $4 \%$ y $5 \%$, respectivamente. Por otro lado, después de la crisis el PIB creció en alrededor del $6 \%$

3 Cálculos propios con base en datos de la ENIGH 2008 y 2010, INEGI.

4 Tasas de crecimiento en comparación con las del año anterior. Datos consultados en el BIE del INEGI. 
pero no se observó una mejora en la situación del mercado de trabajo, ya que la tasa de desocupación permaneció en $6 \%$.

En cuanto al desempleo femenino, cabe resaltar que aumentó fuertemente en el momento más agudo de la crisis. No obstante, a diferencia de la depresión económica de 1994, su tasa fue similar a la de los hombres, después de mostrar un fuerte aumento desde finales de 2007. Para el caso de las mujeres esto pudiera deberse a la incorporación de un mayor número de ellas al mercado de trabajo como una forma de hacer frente a la crisis económica y de poner freno a la caída de los ingresos del hogar; pero también al efecto multiplicador que tuvo la desaceleración del sector manufacturero sobre el resto de los sectores económicos, como los servicios y el comercio, en los cuales tradicionalmente se han ocupado las mujeres.

En el caso de los hombres, las razones del desempleo se encuentran en el tipo de desarrollo económico que ha seguido el país, ya que los sectores en los cuales se han empleado en mayor medida son los más golpeados desde la reestructuración económica de la década de los ochenta, aspecto señalado por Rendón (2003). La manufactura resultó sumamente afectada por la crisis económica de 2008-2009 como consecuencia de la relación que existe con el consumo de la población estadounidense. García y Sánchez (2012) mostraron que las ciudades más afectadas por el desempleo fueron aquellas con una fuerte producción manufacturera. El segundo sector más perjudicado fue el de la construcción, a causa de la retracción de la demanda interna de viviendas.

México siguió las tendencias del empleo observadas en América Latina, pero con repercusiones negativas más profundas (CEPAL \& OIT, 2010). No es de sorprender que el nivel de empleo en la manufactura tuviera una pérdida en su participación en el total de empleos, que disminuyó en tres puntos porcentuales de 2008 a 2009, pues pasó del $28 \%$ al $25 \%$ del total de los ocupados, según sector de actividad en las zonas urbanas. ${ }^{5}$

La tendencia al alza en el desempleo pudiera sugerir una dificultad cada vez mayor para realizar actividades de subsistencia o insertarse en el sector informal de una gran cantidad de personas que no encuentran cabida en empleos protegidos. Pero también pudiera significar que la creación de empleos formales es cada vez menor y que no son suficientes para los crecientes contingentes de población que se incorporan año con año al mercado de trabajo, cuestión que pudo haberse acentuado con la permanencia de las políticas neoliberales y las medidas pasivas que se han seguido en el tema del empleo.

Otro de los indicadores que muestran la situación del trabajo en momentos de crisis económicas es el desenvolvimiento de los empleos asalariados formales (gráfica 2). Los registros del Instituto Mexicano del Seguro Social (IMss) permiten

5 Cálculos propios a partir del sistema de Consulta Interactiva de Datos del INEGI. 
observar que en 2009 hubo una disminución abrupta del número de empleos formales cercana a los 530 mil empleos permanentes, los cuales se recuperarían hasta octubre de 2010. En cuanto a los trabajadores eventuales, se observó una caída de casi 150 mil plazas durante el año de la crisis.

Al comparar las tendencias observadas entre el número de empleos permanentes y el de los temporales se nota que los últimos se recuperaron en menor tiempo y aumentaron de manera más acelerada. Esto podría estar señalando que una de las consecuencias de la crisis es que los empleadores - empresas o gobierno-, una vez que hubo un ambiente económico adecuado para la inversión, decidieron cambiar puestos de trabajo permanentes por eventuales. Lo anterior significaría que muchos de los capitalistas decidieron pasar a los trabajadores los riesgos que implica una crisis, a modo de tener la menor cantidad posible de pérdidas económicas. Al respecto, Lomelí y Murayama (2009) opinaron que la gran pérdida de empleos formales está mostrando que en el país existe un alto grado de flexibilización laboral en términos de despido, pero que se trata de una flexibilidad asimétrica debido a que la gran cantidad de despidos no corresponde al número de puestos de trabajo formales generados en las fases de expansión del ciclo económico.

GRÁFICA 2. Zonas urbanas de México, 1997-2012. Trabajadores asegurados eventuales y permanentes en el IMSs*

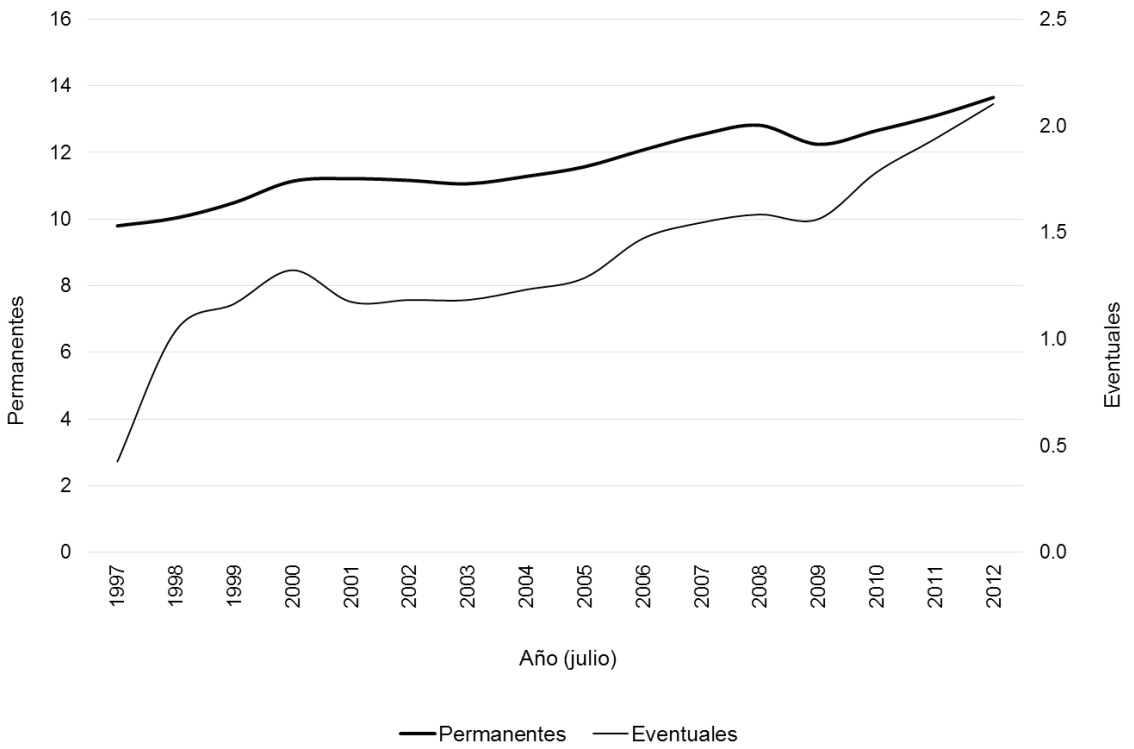

* Datos a julio de cada año. Millones de personas.

Fuente: Elaboración propia a partir de datos de la Secretaría del Trabajo y Previsión Social. 
Por otro lado, había que preguntarse dónde se concentró el mayor número de despidos. Los datos mencionados muestran una pérdida de puestos de trabajo formales. Gran parte de este tipo de empleos se concentra en grandes empresas, que tienen mayores márgenes de maniobra para enfrentar las dificultades económicas. Faltaría estudiar qué pasó con las empresas y los negocios de menor tamaño, que no registran a sus empleados ante el IMSs, por lo que se encuentran más desprotegidos; sin embargo, los datos existentes no lo permiten.

\section{Clase socioocupacional de los hogares, mercado de trabajo y crisis económica}

En el apartado anterior se presentaron brevemente los efectos de la crisis económica sobre el mercado de trabajo en las zonas urbanas del país, en el que se le dio prioridad a los niveles de la población desocupada y del empleo formal. En la presente sección se analizan los cambios observados en el tipo de oferta laboral de los hogares entre 2008 y 2010, a partir de una propuesta de clasificación socioocupacional de las unidades domésticas que participaron en el mercado de trabajo. Dicha propuesta busca captar las desigualdades que se generan en los mercados de trabajo del país y los cambios ocurridos a partir de la implementación de las reformas estructurales, que han dado lugar a condiciones laborales y remuneraciones distintas para la población trabajadora.

Asimismo, se realizó un intento de superar las limitaciones que observó Mora (2004) en distintas investigaciones sobre estratificación y clases sociales que tomaron como válido el supuesto de que el jefe del hogar expresa las características de los miembros de la unidad doméstica e "incurren en una significativa simplificación de la estructura social, al tiempo que soslayan las transformaciones sociales en curso" (p. 11), tales como un aumento de la participación económica femenina y el incremento de perceptores laborales por hogar.

Por lo tanto, para la estratificación propuesta se requiere incluir la composición socioocupacional del hogar en su conjunto debido a que, como lo señala Mora (2004), condiciona su nivel de bienestar el grado de vulnerabilidad y de afectación por los procesos de cambio económico. ${ }^{6}$ En consecuencia, se propone una clasificación de las unidades domésticas que participan en el mercado laboral según las distintas posiciones en el trabajo que tienen sus miembros. ${ }^{7}$

6 Mora (2004) también considera que las características sociodemográficas de los individuos que conforman un hogar tendrán relación con el bienestar y el grado de vulnerabilidad.

7 Para hacer esta clasificación se consideraron las ocupaciones de todos los miembros del hogar que estaban ocupados. También se incluyeron las actividades del segundo empleo, en caso de tenerlo, de todos los ocupados del hogar. 
En primer lugar, se contempló la pertenencia a sectores asalariados o no asalariados, y posteriormente si todos los miembros del hogar son asalariados y si cuentan con protección social o no, para establecer si las unidades domésticas son precarias o protegidas. Estas distinciones se hicieron a partir de que en México desde la década de los setenta se observó que la pertenencia a sectores asalariados y no asalariados ha estado relacionada con un acceso diferencial a recursos, vinculado a un proceso de división social del trabajo y de concentración de riqueza; las retribuciones al trabajo son una de las fuentes de esta desigualdad (García, Muñoz \& Oliveira, 1982; García \& Oliveira, 2003; Pacheco, 2004).

Asimismo, la diferenciación entre precario y protegido se hizo con la finalidad de incluir los cambios que ha sufrido el empleo asalariado en la región latinoamericana a partir de los programas de reestructuración económica. Mora (2010) incluyó en el concepto de trabajo precario únicamente la degradación del empleo en relación con el trabajo asalariado en la fase del capitalismo global.

Se establecieron cuatro clases de hogares, que son: asalariados protegidos, asalariados precarios, no asalariados y mixtos. ${ }^{8}$ Cabe señalar que los hogares de trabajadores (con al menos un ocupado) representaron cerca del $83 \%$ del total en 2008 y 2010. El resto de las unidades domésticas están conformadas por hogares patronales (al menos uno de sus integrantes contrata trabajo asalariado), hogares desocupados (todos sus miembros económicamente activos se encontraban desocupados) y hogares no económicamente activos (ninguno de sus miembros participaba en el mercado laboral).

Las características que se tomaron para la clasificación socioocupacional se presentan en el cuadro $1 .{ }^{9}$

En el cuadro 2 se presenta la distribución de los hogares a partir de la clasificación socioocupacional. Se advirtió que para 2008 predominan las unidades domésticas de tipo asalariado precario con $49.2 \%$, seguidas por los asalariados protegidos con $22.9 \%$, proporciones que permanecieron inalteradas para 2010. Al parecer, a los hogares protegidos el hecho de que sus miembros ocupados contaran con contrato de trabajo y estuvieran inscritos en algún sistema de seguridad social les aseguró la permanencia en el puesto de trabajo y una mayor protección social que al conjunto de los miembros de la unidad doméstica.

8 Únicamente se incluyeron hogares que declararon tener al menos un miembro ocupado.

9 Aunque la vulnerabilidad económica es una dimensión del empleo precario, no se considera el nivel de ingresos del hogar porque una parte del presente artículo está dedicada a observar las diferencias en los niveles de las remuneraciones laborales según la clase socioocupacional de la unidad doméstica, por lo que su inclusión tendría como resultado un argumento circular. 
CUADRO 1. Criterios para definir la clase socioocupacional de los hogares de los trabajadores.

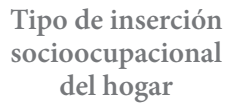

Asalariado

protegido

Asalariado

precario

No asalariado

Mixto

Fuente: Elaboración propia.

\section{Criterios de clasificación}

Todos los ocupados del hogar son asalariados que cuentan con contrato y seguridad social.

Al menos el $75 \%$ de los miembros del hogar reúnen los requisitos para ser cubiertos por la seguridad social, como extensión de esta prestación laboral por parte de los miembros ocupados.

Todos los ocupados del hogar son asalariados y tienen al menos una de estas características:

Al menos un asalariado no tiene contrato.

Al menos un asalariado no tiene seguridad social.

Los asegurados del hogar por parte del trabajo del asalariado representan menos del $75 \%$ de los miembros del hogar.

Todos los ocupados se dedican a actividades no asalariadas:

Trabajadores por cuenta propia.

Trabajadores por cuenta propia que tienen trabajo no remunerado.

Trabajadores no remunerados.

En este tipo de hogar hay trabajadores asalariados y no asalariados.

CUADRO 2. Zonas urbanas de México, 2008 y 2010. Distribución porcentual de los hogares según clase socioocupacional

\section{Clase socioocupacional del hogar}

Asalariado protegido

Asalariado precario

No asalariado

Mixto

Total

\section{8}

22.9

49.2

10.2

17.7

100
2010

22.9

49.3

$11.8^{*}$

$16.0^{*}$

100

* Diferencia estadísticamente significativa, $a=0.05$.

Fuente: Elaboración propia a partir de ENIGH 2008 y 2010, INEGI.

En cuanto a la mayor presencia de hogares de tipo asalariado, ya sea precario o protegido, cabe señalar que en las zonas urbanas de México predomina el empleo asalariado, ya que el $70 \%$ de sus ocupados lo son para 2008 y 2010, aunque solo la mitad de ellos tienen contrato escrito. ${ }^{10}$ Esto se expresó en que la mitad de los hogares tuvieran baja o nula protección otorgada por el empleo, lo cual se privilegió en el

10 Cálculos propios a partir de ENIGH 2008 y 2010, INEGI. 
pasado para hacer extensivos los "beneficios del desarrollo" a las familias (Valencia, 2006). Aunque el proceso de asalarización fue paulatino durante el siglo $\mathrm{xx}$, la protección y los beneficios otorgados por el trabajo no se extendieron al mismo ritmo, por lo que se favoreció a solo una parte de los hogares. También a partir del proceso de precarización del empleo asalariado observado desde la década de los ochenta (Mora, 2010; García, 2011; Pacheco, 2014) puede suponerse que ha afectado a los hogares, pues ha disminuido la proporción de los que se consideran como protegidos.

A partir de la implementación de las reformas estructurales de corte neoliberal, la situación económica de los hogares se ha complicado debido a la falta de creación de empleos de calidad y de la constante pérdida de poder adquisitivo de las remuneraciones laborales (García y Pacheco, 2000; Oliveira y Ariza, 2001; Lozano, 2007; Damián, 2012). A esto hay que agregar efectos de las crisis económicas como el aumento del desempleo y la inestabilidad en los niveles de precios.

Por otro lado, la inexistencia de otro tipo de recursos económicos en muchas unidades domésticas ha provocado que busquen fuentes alternativas de generación de ingresos, como el trabajo por cuenta propia o el establecimiento de un negocio en el que participen los miembros del hogar. Para 2010, esto explicaría en parte el aumento que tuvieron los hogares no asalariados de $10.2 \%$ a $11.8 \%$. Además, se explicaría por una disminución de los hogares de inserción mixta de $17.7 \%$ a $16 \%$. Es probable que, debido al aumento del desempleo, los trabajadores que se encontraban laborando para algún negocio o empresa quedaran desocupados, por lo que el hogar perdería el componente asalariado y obtendría recursos únicamente de los trabajadores por cuenta propia o de negocios propios.

Estos cambios también podrían sugerir que se trató de una respuesta de los hogares para hacerse de ingresos que permitieran la manutención de los mismos. La aparición de una porción de estas actividades es anticíclica, y pueden convertirse en una opción para los hogares. Oliveira (1999) observó que ante la escasez de empleos y la expansión de la oferta de trabajo, la heterogeneidad laboral se manifiesta en la creación de autoempleos, como una estrategia de la fuerza de trabajo.

Sin embargo, es necesario tomar en cuenta que la producción de bienes y servicios generados por medio de actividades no asalariadas se dirige en gran medida hacia sectores medios y populares, que también son golpeados por la crisis, por lo que se tendría que estudiar que tan "exitosa" fue esta medida en el sentido de obtener recursos suficientes para la adecuada reproducción de la fuerza de trabajo. Los datos que se presentan más adelante sobre los ingresos por hora de trabajo de los hogares permitirán contestar parcialmente esta pregunta. 


\section{Los ingresos por trabajo ante la crisis ${ }^{11}$}

Los ingresos por hora de trabajo registraron fuertes afectaciones por la coyuntura económica vivida en el país en 2009 (cuadro 3). ${ }^{12}$ Para el análisis de los ingresos por hora se presentan dos indicadores: la media y la mediana. En cuanto al primero, para el total de los hogares trabajadores la reducción de los ingresos promedio por hora laborada fue de casi $10 \%$. Los que más resintieron la crisis fueron los hogares no asalariados, pues tuvieron una reducción de sus ingresos promedio de $23 \%$, los cuales disminuyeron de 27.4 a 21.0 pesos, mientras que los asalariados protegidos sufrieron una reducción del $6 \%$.

En México es conocido que existe una alta concentración del ingreso corriente de los hogares, situación que también afecta a los ingresos laborales, por lo que la media suele mostrar valores altos, a diferencia de la mediana. En general, se observó que los ingresos laborales medios de los hogares son más altos que los ingresos medianos, lo que confirmaría que este último es un indicador más adecuado, por lo que a continuación se presenta su análisis.

CUADRO 3. Zonas urbanas de México, 2008 y 2010. Media y mediana de los ingresos reales por trabajo por hora según clase socioocupacional del hogar (pesos) (agosto de $2010=100$ )

\begin{tabular}{lcccccc}
$\begin{array}{c}\text { Clase socioocupacional } \\
\text { del hogar }\end{array}$ & \multicolumn{2}{c}{2008} & \multicolumn{2}{c}{2010} & \multicolumn{2}{c}{$\begin{array}{c}\text { Cambio porcentual } \\
\text { 2008-2010 }\end{array}$} \\
& Media & Mediana & Media & Mediana & Media & Mediana \\
\hline Asalariado protegido & 47.6 & 34.0 & $44.8^{*}$ & $30.9^{*}$ & -6 & -9.21 \\
Asalariado precario & 28.0 & 20.2 & $25.9^{*}$ & $17.9^{*}$ & -7.51 & -11.28 \\
No asalariado & 27.4 & 14.7 & $21.0^{*}$ & $13.1^{*}$ & -23.36 & -11.44 \\
Mixto & 25.6 & 18.2 & $22.3^{*}$ & $16.6^{*}$ & -13.17 & -8.91 \\
Total & 32.0 & 21.5 & $29.1^{*}$ & $19.5^{*}$ & -9.2 & -9.45
\end{tabular}

* Diferencia estadísticamente significativa con respecto a 2008, nivel de significancia del 0.05. Prueba U de Mann-Whitney. Fuente: Elaboración propia a partir de ENIGH 2008 y 2010, INEGI.

El total de unidades domésticas de trabajadores tuvieron una pérdida en la mediana de los ingresos de $9.45 \%$ entre ambos años de estudio. Los hogares no asalariados registraron una mayor contracción en la mediana de los ingresos, con un

11 Se incluyen únicamente los ingresos monetarios de los hogares dado que se encuentran en zonas urbanas, por lo que la mayor parte de las necesidades se satisfacen en el mercado y se requiere de dinero para los intercambios mercantiles.

12 Para deflactar los ingresos laborales de los hogares se utilizó el Índice Nacional de Precios al Consumidor y se tomó como periodo base agosto de 2010. 
decremento de $11.44 \%$, seguidos por los hogares asalariados precarios con -11.28 $\%$. Acerca de la mediana, es importante resaltar que la mitad de las unidades estudiadas en 2010 tuvieron ingresos por hora menores de 19.5 pesos.

$\mathrm{Al}$ parecer, los hogares asalariados protegidos tuvieron mayores posibilidades de obtener mejores ingresos por sus labores; a diferencia del resto, que obtuvieron ingresos medios y medianos menores para ambos años de estudio. Esto pudiera sugerir que las actividades no asalariadas en muchas ocasiones son actividades de subsistencia, es decir, que no le permiten al hogar en cuestión acumular, solo obtener ingresos que para la manutención cotidiana.

Los datos mostrarían que, para el caso de los hogares asalariados precarios y protegidos, la crisis agravó la explotación que sufren los trabajadores. Los datos permiten afirmar que hubo un estancamiento en los salarios nominales entre $2008 \mathrm{y}$ 2010, lo que dio lugar a una fuerte reducción de las remuneraciones reales y, por lo tanto, en los ingresos de los hogares. La difícil situación económica por la que atravesó el país y el aumento del desempleo pudieron ejercer presión sobre los asalariados para que aceptaran estas condiciones de trabajo y así mantener sus empleos.

Pacheco (2004) encontró que las políticas de contracción salarial afectaron a gran parte de los trabajadores, pero en ocasiones los trabajadores por cuenta propia mostraron tener alguna ventaja en términos de ingresos por trabajo frente a los asalariados, lo cual puede reflejarse en la dispersión de ingresos que tiene este sector. A esto habría que agregarle que las remuneraciones que reciben los trabajadores deben enmarcarse en la subsistencia de los hogares, es decir, en cuántas personas dependen de ese ingreso. Los datos obtenidos en esta investigación muestran que los hogares no asalariados obtienen ingresos medianos menores que los asalariados protegidos. En este caso se considera que el autoempleo se ha convertido en una alternativa ante la disminución de oportunidades de obtener un empleo formal.

Por otro lado, los trabajadores asalariados están cargando con los costos asociados a una crisis, al no recibir aumentos en sus remuneraciones que reflejen los aumentos en los precios de los bienes que más consumen. Los incrementos que se calculan en el Índice Nacional de Precios al Consumidor (INPC) no están integrando adecuadamente los impactos que han recibido los precios de diversos bienes y servicios de consumo generalizado, por parte de las coyunturas que se están viviendo en la actualidad global. Puesto que la economía mexicana ha estrechado sus lazos con la economía mundial y ha destruido parte de su capacidad productiva, el país no es ajeno a diversos eventos que suceden fuera de las fronteras nacionales, como la crisis financiera. Los grandes capitales trasnacionales tienen mayores oportunidades de sobrevivir; en cambio, las pequeñas y medianas empresas enfrentan muchas dificultades para subsistir y en el peor de los casos desaparecen, y junto con ellas los empleos que han creado. 


\section{Crisis económica y esfuerzo laboral de los hogares}

Para la reproducción material de la fuerza de trabajo, los hogares deben dedicar tiempo a trabajar para el mercado, así como a las tareas domésticas y de cuidado. De acuerdo con las necesidades de cada unidad doméstica, destinan un distinto número de horas a cada una de estas labores. Sin embargo, puede suponerse que, debido al deterioro de los salarios, con el paso del tiempo los hogares han tenido que dedicar un mayor número de horas a ambas actividades. Por un lado, la caída en el poder adquisitivo de las remuneraciones se ha relacionado con un aumento de la participación de un mayor número de miembros del hogar para hacer frente a la reducción de los ingresos. Por otro lado, pudo suceder que parte de los bienes y servicios que podían adquirirse con anterioridad en el mercado ahora deben ser producidos en los hogares, por lo que ambas situaciones han impactado negativamente en sus condiciones de vida.

Para analizar las diferencias existentes en la cantidad de trabajo realizado para el mercado entre hogares, se presenta el número de horas semanales que le dedicaron por medio de dos indicadores: el promedio por hogar y el promedio por ocupado (cuadro 4 ).

En un primer momento, pudiera afirmarse que la oferta de fuerza de trabajo por parte de los hogares no se modificó sustancialmente durante la crisis, aunque se hubiera esperado una reducción de las horas dedicadas al trabajo para el mercado a causa de la disminución de la actividad económica en general. Sin embargo, se observa que las horas totales por hogar siguieron siendo prácticamente las mismas, 81 semanales. Las diferencias entre las unidades domésticas según la clasificación socioocupacional radican en el número de ocupados. ${ }^{13}$

Las unidades domésticas de tipo mixto mostraron una mayor cantidad de miembros y de ocupados, por lo que le destinaron una mayor cantidad promedio de horas al trabajo, 111.2 semanales en 2008, y para 2010 aumentan su oferta laboral en más de cuatro horas. En cambio, en los hogares asalariados precarios se redujo en más de una hora, al pasar de 81.9 a 80.6. En el caso de los hogares no asalariados el cambio no fue estadísticamente significativo.

Para hacer comparaciones sin el efecto del tamaño de hogar, se presentan las horas por ocupado (cuadro 4). Para el total de las unidades domésticas de los trabajadores, el indicador se ubicó por arriba de 45.5 horas semanales en 2008, y aumentó a casi 47 en 2010, en tanto que las asalariadas protegidas presentaron una mayor cantidad de horas, con 47.7 en 2008, cifra que aumentó a 48.9 para 2010. Los hogares no

13 Los hogares mixtos tienen una proporción mayor de tres o más ocupados; al contrario de los no asalariados y de los asalariados protegidos, en los que alrededor de dos tercios tienen solo un ocupado (véase el cuadro del anexo). 
asalariados le dedicaron una menor cantidad de tiempo al trabajo para el mercado en 2008 con 41.4 horas a la semana; sin embargo, fueron los hogares que observaron un aumento más pronunciado en la jornada para 2010, con más de tres horas.

A nivel individual, la información expuesta apuntaría a que el aumento en los dos últimos indicadores mostraría una caída en los niveles de empleo más que proporcional respecto al decremento en la producción. En el caso de los hogares asalariados, tanto precarios como protegidos, pudo suceder que algunas empresas ajustaran sus costos laborales por medio de una gran cantidad de despidos, por lo que las cargas de trabajo restantes recayeron en los trabajadores que conservaron su empleo, lo que implicó un mayor número de horas de trabajo.

CUADRO 4. Zonas urbanas de México, 2008 y 2010. Promedio de horas semanales dedicadas al trabajo para el mercado según clase socioocupacional del hogar

\begin{tabular}{lrccc}
\multicolumn{1}{c}{$\begin{array}{c}\text { Clase socioocupacional } \\
\text { del hogar }\end{array}$} & Por hogar & Por ocupado & Por hogar & Por ocupado \\
\hline Asalariado protegido & 66.8 & 47.7 & $68.0^{*}$ & $48.9^{* *}$ \\
Asalariado precario & 81.9 & 45.9 & $80.6^{*}$ & $47.3^{* *}$ \\
No asalariado & 60.2 & 41.4 & 61.3 & $44.5^{* *}$ \\
Mixto & 111.2 & 43.8 & $115.5^{* *}$ & $44.6^{* *}$ \\
Total & 81.4 & 45.5 & 81.1 & $46.9^{* *}$
\end{tabular}

Diferencia estadísticamente significativa con respecto a $2008:{ }^{*} \alpha=0.1,{ }^{* *} \alpha=0.05$.

Fuente: Elaboración propia a partir de ENIGH 2008 y 2010, INEGI.

En el caso de los no asalariados los datos podrían sugerir que el ajuste se debió más a la caída en la demanda de los bienes y servicios que estos producían. En el caso de tener un negocio de tipo familiar pudieron ampliar los horarios de servicio como forma de atraer una mayor demanda, y en el de los hogares de inserción mixta, un mayor número de trabajadores familiares permitiría repartir la carga laboral extra entre una mayor cantidad de miembros.

Sobre las jornadas laborales se puede afirmar que las condiciones de compra y venta de fuerza de trabajo en tiempos de crisis "inclinan la balanza" a favor de los empresarios. Al hablar sobre el derecho de tiempo para el ocio, Damián (2010) señala que en cualquier sociedad dividida en clases el control del tiempo de los demás es fundamental para la apropiación de la fuente de toda riqueza, es decir, del trabajo humano.

El aumento en las jornadas laborales pudo tener diversas consecuencias en los hogares, principalmente para las mujeres de ellos, quienes es posible que hayan tenido que aumentar su carga global de trabajo; es decir, del tiempo que dedican al tra- 
bajo para el mercado y el destinado al trabajo de cuidado y a las labores domésticas. De la misma forma, habría que preguntarse cuáles fueron los efectos de la crisis económica sobre las horas de trabajo totales de los hogares. Como se mencionó al principio de este apartado, la caída de los ingresos reales de los trabajadores pudo provocar que se tuviera que aumentar el tiempo dedicado a las actividades domésticas, al tener que reemplazar con este tipo de trabajo los bienes o servicios que no pueden adquirir en el mercado.

También es necesario reflexionar acerca de que, dado que las horas de un día son finitas, el alargamiento de la jornada laboral pudo haber tenido consecuencias en la convivencia familiar, pues se destina menos tiempo a ella y al descanso y esparcimiento. Asimismo, como menciona Jusidman (2009), la necesidad de trabajar limita el tiempo que los jóvenes permanecen en la escuela por la dificultad que implica compatibilizar ambas actividades. Y aun permaneciendo en el sistema educativo, le dedican menos horas al estudio, lo que afecta su desempeño y aprendizaje.

Al parecer los datos aquí presentados dan sustento a la idea de García y Oliveira (2003), quienes subrayan que la heterogeneidad laboral en sí misma es pertinente para explicar las diferencias de ingresos entre los trabajadores, lo que también aplicaría para los hogares. Se puede afirmar que las unidades domésticas no asalariadas fueron las más afectadas pues son las que reciben menos ingresos y que, en promedio, registran menos horas de trabajo en comparación con los demás hogares, lo que les hace más propensas a recibir una menor cantidad de ingresos totales.

Como un primer resultado, los datos sobre las horas trabajadas, así como los ingresos por hora de trabajo, podrían sugerir que para los hogares asalariados la disminución de los ingresos reales se dio por el estancamiento de los salarios, que no se ajustaron al aumento de precios que se observaría durante 2009 y 2010 , lo cual provocó una pérdida de su valor real. Para los trabajadores por cuenta propia significó una disminución en el monto de servicios y productos ofrecidos y vendidos y un abaratamiento del valor real de su fuerza de trabajo como estrategia para vender mayores cantidades ante una caída de la demanda como resultado de la crisis.

Ahora bien, se observa que la crisis económica tuvo efectos diferenciales sobre las horas de trabajo y los ingresos por trabajo dependiendo de la clase socioocupacional a la que pertenecía el hogar. Para hacer más evidentes las diferencias, a continuación se presenta un ejercicio que muestra el esfuerzo laboral, medido en horas de trabajo, que debieran realizar las unidades domésticas antes y después de la crisis para acceder a condiciones de vida dignas.

Con este objetivo en mente, para tener un acercamiento a las condiciones de vida se eligió la Canasta Normativa de Satisfactores Esenciales (CNSE), elaborada originalmente por la Coordinación General del Plan Nacional de Zonas Deprimidas y Grupos Marginados (Coplamar), cuya metodología fue mejorada por Boltvinik y Marín (2003). El diseño de la CNSE consideró un conjunto de satisfactores en 
ocho grupos: alimentación, educación, salud, vivienda, vestido y calzado, cultura y recreación, transportes y comunicaciones, presentación personal y otras necesidades, de manera que permitiera "la satisfacción adecuada de la necesidad en un nivel austero, pero digno" (Coplamar, citada en Boltvinik y Marín, 2003). ${ }^{14}$ Una de sus ventajas es que permite el cálculo de una cesta para cada tipo de hogar ya que se puede incluir la composición por edad y sexo, mediante el indicador de adulto equivalente (AE) ${ }^{15}$ su tamaño y las economías de escala.

En el ejercicio se calculó el valor de una canasta para un hogar promedio según cada una de las clases socioocupacionales, por lo que los datos necesarios se presentan en el cuadro 5. La media del tamaño y de los AE por hogar solo se muestra para 2010 porque no se observaron variaciones entre los años de estudio.

CUADRO 5. Zonas urbanas de México, 2008 y 2010. Horas semanales promedio de trabajo para el mercado requeridas para la adquisición de la CNSE según clase socioocupacional del hogar y tipo de ingreso

\begin{tabular}{lrrrrr}
\multicolumn{1}{c}{ Indicadores } & $\begin{array}{c}\text { Asalariado } \\
\text { protegido }\end{array}$ & $\begin{array}{c}\text { Asalariado } \\
\text { precario }\end{array}$ & $\begin{array}{c}\text { No } \\
\text { asalariado }\end{array}$ & Mixto & Total \\
\hline Tamaño promedio de hogar 2010 & 3.50 & 4.10 & 3.30 & 4.70 & 4.00 \\
Promedio adultos equivalentes & 2.80 & 3.30 & 2.70 & 3.80 & 3.20 \\
Valor monetario cNSE 2008 & $2,918.91$ & $3,296.60$ & $2,829.93$ & $3,674.29$ & $3,224.42$ \\
Valor monetario cNSE 2010 & $2,963.39$ & $3,347.22$ & $2,873.01$ & $3,731.04$ & $3,273.86$ \\
Horas semanales con Ih-medio 2008 & 61.32 & 117.74 & 103.28 & 143.53 & 100.76 \\
Horas semanales con Ih-medio 2010 & 66.15 & 129.24 & 136.81 & 167.31 & 112.50 \\
Horas semanales con Ih-mediano 2008 & 85.85 & 163.20 & 192.51 & 201.88 & 149.97 \\
Horas semanales con Ih-mediano $2010^{3}$ & 95.90 & 187.00 & 219.31 & 224.76 & 167.89
\end{tabular}

1 Valor semanal de la canasta a pesos constantes de agosto de 2010.

2 Ih-medio: ingreso por hora medio.

${ }^{3} \mathrm{Ih}$-mediano: ingreso por hora mediano.

Fuente: Elaboración propia a partir de ENIGH 2008 y 2010, INEGI.

14 La forma general de la canasta es: $\mathrm{CNSE}=\mathrm{A}+\mathrm{B}(\mathrm{P})+\mathrm{C}(\mathrm{AE})$, donde:

$\mathrm{A}=$ costo de los bienes familiares fijos (economías a escala).

$\mathrm{B}=$ costo de los bienes familiares variables.

$\mathrm{C}=$ costo del adulto hombre.

$\mathrm{P}=$ número de integrantes del hogar.

$\mathrm{AE}=$ número de adultos equivalentes en el hogar.

15 Para tomar en cuenta las diferencias de edad y sexo de los integrantes del hogar se utilizó el método de adulto equivalente, el cual se define como un parámetro que mide dichas diferencias en cuanto a requerimientos calóricos y proteínicos, basándose en los requerimientos de un hombre adulto, el cual vale 1. Es decir, se otorgan ponderadores distintos a los miembros del hogar según su sexo y edad. 
Como se puede advertir, los indicadores varían según la clase socioocupacional de los hogares, lo que puede indicar una asociación entre su número de integrantes y las actividades que realizan en el mercado de trabajo. Las diferencias por AE también muestran las distintas necesidades materiales que existen entre las unidades domésticas según su estructura, es decir, su composición por edad y sexo.

Las unidades domésticas mixtas tienen un tamaño cercano a cinco miembros, lo cual pudiera sugerir que un hogar con un mayor número de ellos tiene mayores probabilidades de ofertar trabajo de distintos modos, es decir, tanto de manera asalariada como no asalariada. Además, la combinación de actividades puede tratarse de una estrategia para obtener mayores ingresos a causa de una mayor cantidad de necesidades debido a un mayor tamaño. Las unidades domésticas más pequeñas fueron las no asalariadas, con un promedio de 3.3 miembros.

Ahora, el valor de la CNSE para el total de las unidades domésticas de los trabajadores fue de 3,224.42 pesos reales a la semana en 2008, y aumentó en alrededor de 50 pesos para 2010. Se trata de un incremento moderado en términos reales si se considera que fue una época de crisis. Lo anterior pudiera explicarse porque la inflación general se mantuvo relativamente baja durante el periodo estudiado.

El siguiente paso fue calcular el esfuerzo laboral que implicaría para los hogares satisfacer sus necesidades por medio de sus ingresos laborales, por lo que se dividió el valor monetario de la CNSE entre el ingreso medio y el mediano por hora de trabajo, lo que dio como resultado una determinada cantidad de horas. De esta forma, se puede tener una mirada de los posibles efectos de la crisis económica de 2008-2009 en el poder adquisitivo de los hogares y su posibilidad de adquirir el conjunto de bienes y servicios necesarios para la satisfacción de sus necesidades.

En un primer momento se puede afirmar que la crisis financiera ocurrida a nivel mundial tuvo como efecto un deterioro en las condiciones de vida de los hogares de los trabajadores, el cual se manifestó en un aumento de horas que deberían dedicarle al trabajo para el mercado. Si se toma el ingreso medio por hora, para cubrir el costo monetario de los satisfactores básicos hubieran tenido que incrementar su jornada laboral en 12 horas semanales. Para los trabajadores de ingreso mediano se tendría que aumentar de 150 a 168 horas, lo que representaría 18 horas más por semana.

Si se considera el ingreso mediano por hora, los hogares no asalariados y los mixtos son los que requieren una mayor cantidad de horas de trabajo para el mercado con el fin de satisfacer sus necesidades, que es superior a las 190 horas en 2008 y a las 220 para 2010. Estos datos denotan las diferencias de ingreso que observan los hogares no asalariados y los hogares mixtos con respecto a los demás; puesto que los primeros son los que reciben remuneraciones laborales más bajas, un tamaño de hogar menor no significa un menor esfuerzo laboral.

En el caso contrario se encuentran los hogares asalariados protegidos, que debieran dedicar una menor cantidad de horas trabajadas para adquirir la CNSE, lo que 
representaría, en promedio, menos de la mitad del tiempo que el resto de las unidades domésticas deberían laborar, tanto con el ingreso medio como con el mediano.

Como último punto, para dar una mayor claridad a lo que significa el esfuerzo laboral que debieran realizar los hogares para su manutención, se presenta el número de ocupados que se requerirían para conseguir los ingresos necesarios para comprar la CNSE (gráfica 3). Este cálculo se hizo tomando como base una jornada semanal por ocupado de 48 horas, es decir, seis días de trabajo por uno de descanso.

GRÁFICA 3. Zonas urbanas de México, 2008 y 2010. Número de personas que debieran laborar por hogar según clase socioocupacional

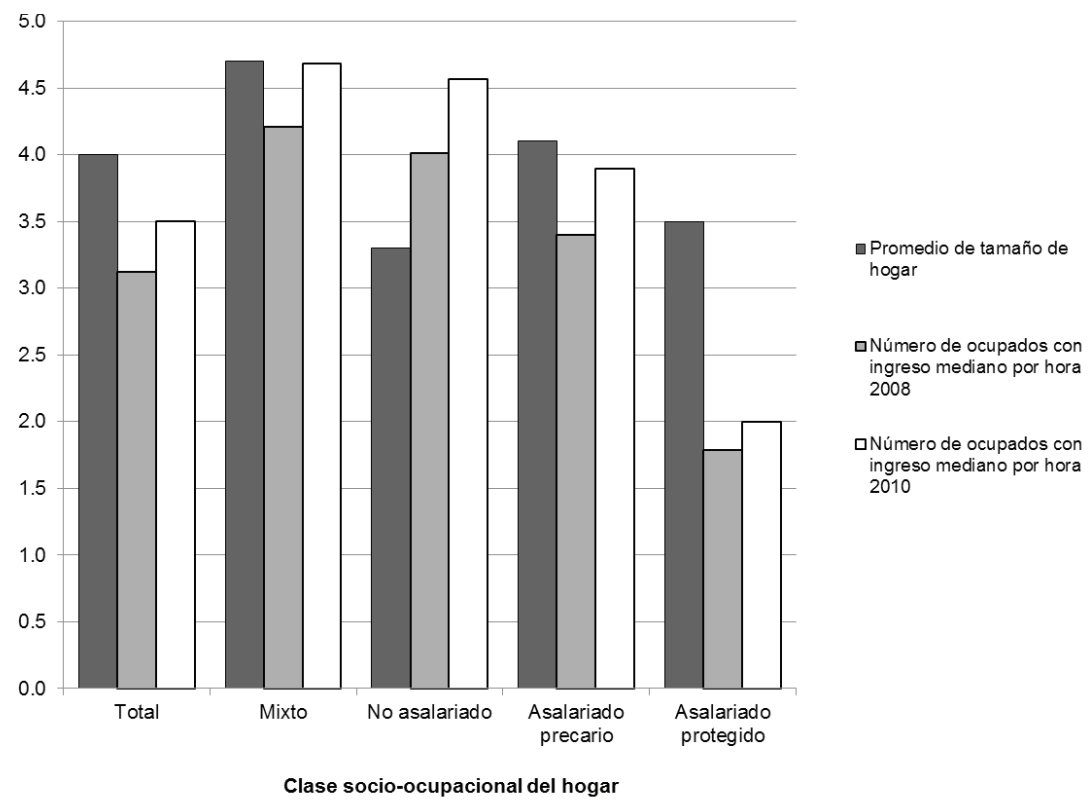

Fuente: Elaboración propia a partir de la ENIGH 2008 y 2010, INEGI.

Como ya se mencionó, el ingreso mediano por hora es un indicador más adecuado que el ingreso medio para el análisis de los ingresos en México debido a que no es afectado por valores extremos. Con base en este indicador, se observó que para 2008 el total de hogares hubiera tenido que ocupar a un poco más de tres miembros para obtener ingresos equivalentes a la CNSE, y aumentó a 3.6 ocupados para 2010.

Las unidades domésticas no asalariadas se encontraron en una situación más inestable que el resto. En 2008, al menos el 50 \% de ellas debieron ocupar cerca de 4 personas, cantidad que aumentó a 4.7 después de la crisis para obtener los ingresos equivalentes al costo de la CNSE. En tanto, son los hogares asalariados protegidos 
los que mostraron mejores niveles de vida porque necesitaban una menor cantidad de ocupados y un menor aumento de 2008 a 2010 (de 1.8 aumentó a 2). Los hogares asalariados precarios pasaron de 3.5 a casi 4 ocupados, mientras que los mixtos aumentaron en 0.5 ocupados al pasar de 4.2 a 4.7 .

Las diferencias en la cantidad de ocupados que requieren las unidades domésticas para generar los ingresos suficientes para satisfacer sus necesidades materiales se deben a una combinación entre el tamaño del hogar y los ingresos medianos generados según la clase socioocupacional. Como ejemplo, aunque necesitan un número de ocupados similar las unidades domésticas mixtas y las no asalariadas, es un hecho que en promedio las segundas presentan unas condiciones de vida más deterioradas debido a que tienen ingresos medianos por hora más bajos y a que tendrían una fuerza de trabajo potencial menor por tener un tamaño promedio de hogar más pequeño (3.3 personas, como se mostró en el cuadro 5).

De igual forma, ambas variables muestran las desigualdades que enfrentan los hogares en el mercado de trabajo, lo que se traduce en distintas condiciones de vida. En este sentido, las unidades asalariadas protegidas son las que mayores posibilidades tendrían de satisfacer sus necesidades materiales. Por lo tanto, el proceso de precarización del empleo asalariado tiene su reflejo en los niveles de vida de los hogares. Mientras que aquellos que gozan de un empleo con prestaciones laborales y contrato de trabajo también acceden a mayores remuneraciones laborales y a servicios de salud, el resto de los trabajadores tienen que hacerle frente a la manutención diaria de sus hogares únicamente con los ingresos que reciben por la venta de su fuerza de trabajo, lo que los ubica en una situación de vulnerabilidad permanente al no tener recursos monetarios con que hacer frente a eventos desfavorables.

Los datos presentados evidencian la imposibilidad real que tienen una gran parte de los hogares de los trabajadores para acceder a los bienes y servicios para su reproducción cotidiana. Por un lado, el tamaño de hogar impone una restricción al uso de una mayor cantidad de fuerza de trabajo, lo que permitiría suplir los bajos ingresos que se generan en el mercado de trabajo. Pero lo anterior implicaría utilizar al total de los miembros del hogar, incluyendo niños y adultos mayores, es decir, población que no está en condiciones de participar económicamente.

Asimismo, se requiere considerar la composición por sexo y edad de las unidades domésticas, lo que incidiría en las distintas actividades que realizan sus integrantes, como asistir a la escuela o dedicarse a las labores domésticas. Por lo tanto, además del tamaño existen otras cuestiones que restringen el uso de la potencial fuerza de trabajo. Por ejemplo, se encuentra el hecho de que la participación de las mujeres depende también del estrato socioeconómico al cual pertenecen. Pacheco (2010) señala que para las mujeres que pertenecen a los sectores bajos de la estructura social la falta de oportunidades constituye un ámbito que reproduce patrones tradicionales de participación económica, lo cual inhibe la entrada de las mujeres en el mercado de trabajo. 
Finalmente, la crisis económica trajo como consecuencia un descenso en los ingresos laborales y en las condiciones de vida para el total de los hogares, lo cual se reflejó en un aumento del número de ocupados que deberían tener las unidades domésticas para mantener los niveles de vida alcanzados antes de la crisis. Sin embargo, aunque el ejercicio presentado se utilizó para ejemplificar el esfuerzo que debieran realizar los hogares, en realidad no tienen las posibilidades de ampliar en forma extensiva su fuerza de trabajo debido a que tienen un número limitado de integrantes; además es posible que estos no puedan descuidar las actividades que realizan para dedicarse al trabajo para el mercado. Por lo tanto, la crisis económica de 2008-2009 tuvo como consecuencia un descenso inevitable en las condiciones de vida de los hogares de las zonas urbanas de México.

\section{Reflexiones finales}

Esta investigación tuvo como objetivo observar y analizar los cambios en los ingresos laborales de los hogares de los trabajadores en las zonas urbanas de México a partir de la irrupción de la crisis financiera de 2008-2009, así como el esfuerzo laboral que tendrían que realizar las unidades domésticas para mantener sus niveles de vida. Se observó que este evento estructural adverso tuvo un fuerte impacto en la producción del país y que se trasladó a la economía de los hogares por medio del desempleo y la reducción de las remuneraciones reales por trabajo.

Sobre los ingresos laborales es posible afirmar que existió una caída en términos reales, aunque el efecto de la crisis no fue igual para todos los hogares de los trabajadores. Los asalariados, tanto precarios como protegidos, sufrieron una reducción de sus ingresos a raíz de un estancamiento en los ingresos nominales por hora que recibieron los miembros de los hogares que estuvieron ocupados, por lo cual se puede afirmar que los mecanismos de contención salarial funcionaron con más fuerza durante la crisis.

Así mismo, se puede señalar que aumentó la explotación de los trabajadores en general en la economía de las zonas urbanas, lo cual se expresó en un mayor número de horas semanales dedicadas al trabajo para el mercado. Mientras que para los trabajadores no asalariados se trató de una autoexplotación, para los asalariados significó una mayor cantidad de horas de trabajo impago. $Y$ aunque para algunos asalariados el contar con un empleo protegido implicó mantener su puesto durante la crisis económica, no se salvaron de una mayor precarización laboral, manifestada en jornadas laborales más largas. Lo anterior pudo tener implicaciones en las actividades que realizan las unidades domésticas para su reproducción cotidiana, además de una sobrecarga de tareas, tanto domésticas como remuneradas. 
En cuanto al esfuerzo laboral que tendrían que realizar los hogares, se mostró que tendrían que dedicarle un mayor número de horas de trabajo remunerado, así como tener un mayor número de ocupados. Sin embargo, tienen que hacer frente a restricciones materiales reales, como un número reducido de integrantes del hogar, además de limitaciones de orden social tales como los roles que se les asignan a los individuos en función de su sexo y su edad, como asistir a la escuela o dedicarse a las tareas domésticas.

Debido a estas restricciones, es muy probable que las consecuencias de la crisis económica de 2008-2009 sobre los ingresos laborales hayan traído consigo un deterioro general de las condiciones de vida de los hogares de los trabajadores. Es pertinente preguntarse hasta cuándo los salarios podrán recuperar el valor real que perdieron durante el periodo de la crisis. La lenta recuperación de la economía mexicana pone de manifiesto un escenario difícil para que esto suceda, por lo cual se acumulan dos años más a la tendencia de la pérdida del poder adquisitivo de los salarios iniciada a mediados de la década de los setenta del siglo pasado.

\section{ANEXO}

CUADRO A.1. Zonas urbanas de México, 2008 y 2010. Distribución porcentual de los hogares según inserción socioocupacional y número de ocupados

\begin{tabular}{|c|c|c|c|c|c|c|c|c|}
\hline \multirow{2}{*}{$\begin{array}{l}\text { Inserción socioocupa- } \\
\text { cional del hogar }\end{array}$} & \multicolumn{4}{|c|}{2008} & \multicolumn{4}{|c|}{2010} \\
\hline & 1 & 2 & 3 o más & Total & 1 & 2 & 3 o más & Total \\
\hline Asalariado protegido & 64.4 & 29.6 & 5.9 & 100 & 64.5 & 30.3 & 5.2 & 100 \\
\hline Asalariado precario & 46.4 & 34.8 & 18.8 & 100 & $49.0^{* *}$ & 35.4 & $15.6^{* *}$ & 100 \\
\hline No asalariado & 68.4 & 21.6 & 10.0 & 100 & 70.5 & 23.7 & $5.9^{* *}$ & 100 \\
\hline Mixto & 6.3 & 52.4 & 41.3 & 100 & $4.8^{\star *}$ & 51.4 & $43.7^{*}$ & 100 \\
\hline Total & 45.7 & 35.4 & 18.9 & 100 & $48.0^{* *}$ & 35.4 & $16.6^{\star *}$ & 100 \\
\hline
\end{tabular}

Diferencia estadísticamente significativa con respecto a 2008, ${ }^{*} \alpha=0.1,{ }^{* *} \alpha=0.05$.

Fuente: Elaboración propia a partir de ENIGH 2008 y 2010, INEGI.

\section{Referencias}

Boltvinik, J. \& Marín, A. (2003). La canasta normativa de satisfactores esenciales de la Coplamar. Génesis y desarrollos recientes. Comercio Exterior, 53(5), 473-484.

Boltvinik, J. (2010). La visión marxista de la crisis y la gran crisis del siglo XXI. En Boltvinik, J. (coord.), Para comprender la crisis capitalista mundial actual. México: Fundación Heberto Castillo Martínez, A.C.

Cámara, S. \& Mariña, A. (2010). Naturaleza y perspectivas de la actual crisis: una caracterización marxista de largo plazo. Política y Cultura, 34, 7-31. 
Comisión Económica para América Latina y el Caribe (CEPAL) (2009). Balance preliminar de las economías de América Latina y el Caribe. Chile: CEPAL.

Comisión Económica para América Latina y el Caribe (CEPAL) \& Organización Internacional del Trabajo (огт) (2010). Coyuntura laboral en América Latina y el Caribe. Boletín CEPAL/OIT, 3.

Cortés, F. \& Rubalcava R., M. (1991). Autoexplotación forzada y equidad por empobrecimiento. México: El Colegio de México.

Damián, A. (2010). El derecho al tiempo para el ocio. En Valencia, E. (coord.), Perspectivas del universalismo en México. México: Fundación Konrad Adenauer, Instituto Tecnológico y de Estudios Superiores de Occidente, Universidad Iberoamericana León, Universidad de Guadalajara.

Damián, A. (2012). Crisis, empleo y pobreza. En Garza, E. de la (coord.), La situación del trabajo en México 2012. México: Universidad Autónoma Metropolitana-Iztapalapa, Plaza y Valdés Editores.

Dumenil, G. \& Levy, D. (2011). The crisis of neoliberalism. Estados Unidos: Harvard University Press.

Estrada, M. (1999). En el límite de los recursos. El efecto de la crisis de 1995 en familias de sectores populares urbanos. En Estrada, M. (coord.), 1995. Familias en la crisis. México: Centro de Investigaciones y Estudios Superiores en Antropología Social, Consejo Nacional de Ciencia y Tecnología.

García, B. (2011). Las carencias laborales en México: conceptos e indicadores. En Pacheco, E. \& Garza, E. de la (coords.), Trabajos atípicos y precarización del empleo. México: El Colegio de México, Centro de Estudios Demográficos, Urbanos y Ambientales.

García, B., Muñoz, H. y Oliveira, O. (1982). Hogares y trabajadores en la Ciudad de México. México: El Colegio de México, Universidad Nacional Autónoma de México.

García, B. \& Oliveira, O. (2003). Trabajo e ingresos de la mano de obra familiar en el México metropolitano. En Garza, E. de la (coord.), La situación del trabajo en México. México: Instituto de Estudios del Trabajo-Universidad Nacional Autónoma de México, Plaza y Valdés Editores.

García, B. \& Pacheco, E. (2000). Esposas, hijos e hijas en el mercado de trabajo de la Ciudad de México en 1995. Estudios Demográficos y Urbanos, 43, 35-63.

García, B. \& Sánchez, L. (2012). Trayectorias del desempleo urbano en México. Revista Latinoamericana de Población, 6(10), 5-30.

Guillén, A. (2009). Crisis global: de las hipotecas a la recesión generalizada. Ola Financiera, 2, 60-96.

Instituto Nacional de Geografía y Estadística (INEGI) (2008). Base de microdatos de la Encuesta Nacional de Ingresos y Gastos de los Hogares 2008. Aguascalientes: INEGI.

Instituto Nacional de Geografía y Estadística (INEGI) (2010). Base de microdatos de la Encuesta Nacional de Ingresos y Gastos de los Hogares 2010. Aguascalientes: INEGI. 
Jusidman, C. (2009). Desigualdad y política social en México. Revista Nueva Sociedad, 220, 190-206.

Lomelí, L. \& Murayama, C. (2009). México frente a la crisis: hacia un nuevo curso de desarrollo. Economía, 6(18), 7-60.

Lozano Arredondo, L. (2007). México: el salario y la necesidad de un proceso de amplia restauración de su poder adquisitivo. En Calva, J. L. (coord.), Agenda para el desarrollo (vol. 11, Empleo, ingreso y bienestar). México: Cámara de Diputados Lx Legislatura, Universidad Nacional Autónoma de México, Miguel Ángel Porrúa.

Mariña, A. (2012). La crisis mexicana de 2008-2010 en perspectiva histórica y en el contexto de la crisis mundial. En García Castro, M. B. (coord.), Estudios sobre México en la crisis mundial: escenario nacional tras dos décadas de apertura y desregulación. México: Universidad Autónoma Metropolitana-Azcapotzalco.

Mora, M. (2004). Visión crítica del vínculo entre jefatura de hogar, estratificación social y análisis de clase. Revista de Ciencias Sociales, 105, 11-24.

Mora, M. (2010). Ajuste y empleo. La precarización del trabajo asalariado en la era de la globalización. México: Centro de Estudios Sociológicos-El Colegio de México.

Ocampo, J. A. (2009). Impactos de la crisis financiera mundial sobre América Latina. Revista de la CEPAL, 97, 9-32.

Oliveira, O. (1999). Políticas económicas, arreglos familiares y perceptores de ingresos. Demos: Carta demográfica de México, 12, 32-33.

Oliveira, O. \& Ariza, M. (2001). Transiciones familiares y trayectorias laborales femeninas en el México Urbano. En Gomes, C. (comp.), Procesos sociales, población y familia. Alternativas teóricas y empíricas en las investigaciones sobre vida doméstica. México: Facultad Latinoamericana de Ciencias Sociales, Editorial Porrúa.

Ortiz, E. (2012). Los nuevos escenarios económicos de la crisis. En Garza, E. de la (coord.), La situación del trabajo en México 2012. México: Plaza y Valdés Editores, Universidad Autónoma Metropolitana-Iztapalapa.

Pacheco, E. (2004). Ciudad de México, heterogénea y desigual. Un estudio sobre mercado de trabajo. México: Centro de Estudios Demográficos y de Desarrollo Urbano-El Colegio de México.

Pacheco, E. (2010). Arreglos familiares y división del trabajo en el hogar: familias con doble proveedor. En Secretaría de Salud (ssa) (coord.), Procesos y tendencias poblacionales en el México contemporáneo: una mirada desde la ENADID 2006. México: Secretaría de Salud, Centro Nacional de Equidad de Género y Salud Reproductiva, Dirección General de Información en Salud, Centro Regional de Investigaciones Multidisciplinarias-Universidad Nacional Autónoma de México.

Pacheco, E. (2014). El mercado de trabajo en México a inicios del siglo xxi. Heterogéneo, precario y desigual. En Guadarrama, R., Hualde, A. \& López, S. (coords.), Dinámicas, transformaciones y significados de la precariedad. Un estudio en tres ocupaciones. En prensa. 
Pérez Akaki, P. (2010). Efectos esperados de la crisis económica actual en las remesas. Perspectivas Sociales $=$ Social Perspectives, 12(1), 11-42.

Rendón, T. (2003). Trabajo de hombres y trabajo de mujeres en el México del siglo xx. México: Programa Universitario de Estudios de Género, Centro Regional de Investigaciones Multidisciplinarias-Universidad Nacional Autónoma de México.

Tuirán, R. (1993). Estrategias familiares de supervivencia en épocas de crisis: el caso de México. En Comisión Económica para América Latina y el Caribe (CEPAL) (coord.), Cambios en el perfil de las familias: la experiencia regional. Chile: CEPAL.

Valencia, E. (2006). La política social de Vicente Fox: contexto histórico y balance. Asian Journal of Latinoamerican Studies, 19(1), 81-99. 